\title{
$\widehat{A}$ Madridge \\ madridge Journal of Nursing \\ PUBLI:
}

Research Article

Open Access

\section{The Impact of Duration of Sleep on Academic Performance in University Students}

\author{
Heather Raley, Jessica Naber*, Summer Cross, and Michael Perlow \\ Department of Nursing, School of Nursing and Health Professions, Murray State University, Murray, USA
}

\section{Article Info}

\author{
*Corresponding author: \\ Jessica Naber \\ Assistant Professor \\ Department of Nursing \\ School of Nursing and Health Professions \\ Murray State University \\ Murray, USA \\ Tel. 502-541-3011 \\ E-mail: jnaber@murraystate.edu
}

Received: May 31, 2016

Accepted: June 28, 2016

Published: July 4, 2016

Citation: Raley HR, Naber JL, Cross S, Perlow MB. The Impact of Duration of Sleep on Academic Performance in University Students. Madridge J Nurs. 2016; 1(1): 11-18.

doi: 10.18689/mjn-1000103

\section{Copyright: @ 2016 The Author(s). This work is licensed under a Creative Commons Attribution 4.0 International License, which permits unrestricted use, distribution, and reproduction in any medium, provided the original work is properly cited.}

Published by Madridge Publishers
Keywords: Sleep; Immune system functioning; Mood; Academic performance.

\section{Introduction}

University students report significantly worse quality of sleep, including inconsistent sleep schedules and sleep deprivation, than the general population. They also suffer from a greater amount of daytime sleepiness than the general population. Students also report significantly fewer total hours of sleep each night than the daily-recommended amount, for their age group, to promote normal cognitive functioning. Students tend to sacrifice sleep to participate in social and academic commitments contributing to constantly changing sleep routines and poor sleeping habits. All of these factors may affect aspects of the average university student's life including mood, immune system function, and even substance abuse (Orzech, Salafsky, \& Hamilton, 2011).

The focus of this study was to assess the student's ability to perform academically in relation to his or her quality of sleep. Despite the fact that a vast amount of time in adolescence is spent learning and improving education, there is a large gap in research examining the relationship of sleep and academic functioning and performance (Shochat et al., 2014).There is a lack of knowledge of very simple modifications in sleep hygiene to improve the quality of sleep in university students, and subsequently, their overall academic performance.

The purpose of this study was to answer the following research question: Does duration of sleep affect univerity students' overall academic performance?A questionnaire was developed to determine sleep habits, subjective perceptions of one's own sleep quality, and any factors that affect the management of sleep and academic performance in university students. The hypothesis was that students who have a longer duration of sleep will have a better academic performance demonstrated by a higher grade point average (GPA)The researcher proposed to investigate the need for implementing a sleep education program component to improve the overall quality of sleep in university students.

\section{Review of Literature}

Shochat et al. (2014) propose that despite the fact that a vast amount of time in adolescence is spent learning and improving education, there is a large gap in research examining the relationship of sleep to academic functioning and performance. Brown et al. (2006), Chen et al.(2014), and Hershner and Chervin (2014) suggest that there is a connection between quality of sleep and students' academic performance. Giannotti et al. (2002) propose health concerns that can present in sleep-deprived students, which include depression, fatigue, and difficulties with attention, concentration, decisionmaking, learning, and facilitating memories. Orzech et al. (2011) discuss how daytime sleepiness results in decreased attention and concentration, which negatively impacts 
learning and memory recall in students. Chen et al. (2014) states that many different variables in the students' sleep environment, such as intrusive light or noise, may have an effect on their quality of sleep. The sleep duration the student is able to obtain determines the amount of rapid eye movement (REM)sleep cycles, memory consolidation and learning the student receives (Hershner \& Chervin, 2014). Milner and Cote (2008) and Gomes et al. (2011) discuss how sleep schedule irregularity and napping can affect student's quality of sleep by delaying the body's natural circadian rhythm and homeostatic sleep drive.

Finally, Brown et al. (2006) discuss how reversing the lack of knowledge regarding behaviors and lifestyle choices that hinder or promote sleep is promising because the distribution of the information can improve students sleeping habits. Hershner and Chervin (2014) and Brown et al. (2006) agree that a sleep education program should be implemented to improve sleeping habits and hygiene of students.

\section{Methods}

\section{Sample}

The study was conducted at a midsize, rural, public university in the southern United States between the dates of 10 February and 10 March 2015. Institutional Review Board (IRB) approval was obtained, and the study included a systematic convenience sample of students from all student classification levels (undergraduate freshman, sophomore, junior, senior; and graduate) of the student population currently enrolled in university courses. Inclusion criteria included the following: (i) age over 18 years; (ii) student currently enrolled in courses at the university.

\section{Instrument}

The questionnaire (see appendix) was produced reflecting background data from research reported in the review of literature. A panel of doctorally prepared nurse faculty evaluated the instrument used for data collection and provided content validity to the questionnaire. Each participant was asked to complete a self-administered online survey that included the collection of demographic information such as gender, age, and ethnicity. The questionnaire included 19 questions consisting of a sleep profile and an academic profile, assessing quality of sleep and academics. The sleep quality profile encompassed sleep duration, sleep environment variables, daytime sleepiness, and the use of sleep remedies. The academic profile encompassed the number of enrolled courses, and the current cumulative grade point average (GPA) which was broken down in to four categories: GPA of 3.50 or higher (Mostly As), GPA of 3.00 to 3.49 (Mostly Bs), GPA of 2.50 to 2.99 (Some Bs and (s), GPA of 2.00 to 2.49 (Mostly Cs). The questionnaire also included a question as to whether the participants believed they were receiving an adequate amount of sleep each night and if they believed they were suffering from a sleep disorder. The responses to these questions were organized categorically into the options of "yes" or "no." [1-5].
Question 6, which read, "When do your classes normally begin each day?" was omitted from the data analysis because $55.7 \%$ of respondents did not respond in part, or skipped this question altogether. Since the students had to indicate on one drop down menu when their classes began for multiple days of the week, the participants may have found it difficult to answer this question.

\section{Procedures}

The recipients of the email (residential college directors) were asked to electronically distribute the online selfadministered questionnaire to their constituents. The constituents included the following: honors program students, students taking introductory psychology courses, and students affiliated with residential colleges. The data collection was conducted electronically for convenience, allowing students of all classifications, majors, and type of residence to complete the questionnaire. The students were invited to complete the survey on their own time before the deadline to close the questionnaire on 10 March 2015. The potential participants were provided with a cover letter explaining the purpose of the questionnaire, inclusion criteria, that information collected would remain anonymous, that participation was voluntary, and that there was no penalty for withdrawal of participation in the study. The participant's completion and return of the questionnaire indicated voluntary consent to participate in this research study, as mentioned in the cover letter.

\section{Statistical Analysis}

Data were entered and analyzed using a computer program, R commander (Rcmdr), which is a platformindependent basic-statistics GUI (graphical user interface) for $R$ programming language (Fox, 2005). This program was used to analyze the sample demographics and answers to the questionnaire. Tests for group trends used $\boldsymbol{\chi}^{\mathbf{2}}$ test for independence to compare categorical data results from two separate questions. A one-way analysis of variance (ANOVA) was used to test for significance in difference of means for normally distributed numerical data of independent groups. To dichotomize the scores, the groups of participants taking less than or equal to three courses were consolidated, because it contained less than $5 \%$ of the total sample. Statistical significance was denoted by $P$ values $<0.05$.

\section{Results}

A total of 168participants completed the online questionnaire. Eight participants were excluded in the final report of data due to incompletion of the questionnaire. Two participants' responses were withdrawn from the final report of data due to the participants being under age 18. One hundred and fifty-eight participants were included in the final data collection $(n=158)$. The majority of the participants were female $(n=135,85 \%)$ with fewer male participants $(n=23$, $15 \%$ ).The participants' student classification distribution were as follows: 19 freshmen, 41 sophomores, 37 juniors, 50 seniors, and 11 graduate students. The sample's ethnicity distribution 
included the following: 147 White/Caucasian, 4 Black/African American, 1 Asian or Pacific Islander, 2 Hispanic or Latino, 2 American Indian or Alaskan Native, and 2 participants preferred not to answer. The mean age of the participants was 21.38years with a standard deviation of 4.67 years.

No significant difference in the quality of sleep by gender $(p=0.143)$ was discovered, illustrating that students of both genders experience about the same quality of sleep. No significant differences in the quality of sleep by student classification ( $p=0.821)$ were discovered, illustrating that seniors, juniors, sophomores, freshmen and graduate students experience the same quality of sleep. The study sample consisted of almost entirely white/Caucasian participants $(n=147,93 \%)$. Therefore, data across genders and ethnicity were analyzed together. The student classification level distribution was relatively even throughout, including: $(n=19$, $12 \%)$ freshmen, $(n=41,25.9 \%)$ sophomores, $(n=37,23.4 \%)$ juniors, $(n=48,30.4)$ and seniors, and $(n=13,8.2 \%)$ graduate students [5-10].

Students of different classification levels reported a significant difference in duration of sleep on a typical night including: freshman with 7.28 hours, sophomore with 7.05 hours, junior with 6.83 hours, senior with 6.38 hours, graduate with 6.08 hours; with a negatively correlated trend that the duration of sleep on a typical night in a university student decreases as classification (education) level increases. The distributions of the amount of courses participants are currently enrolled in are as follows: $(n=20,12.7 \%)$ in "1-3 courses," ( $n=49,31 \%)$ in "4 courses," and $(n=89,56.3 \%)$ in " $5+$ courses." The participants with fewer courses reported less average hours of sleep each night: 1-3 courses had a mean of 6.40 hours, 4 courses mean of 6.74 hours, $5+$ courses had a mean of 6.82 hours.

The majority of participants had a GPA in the highest category of "3.5 or higher," and the responses were as follows: $(n=91,57.6 \%)$ with a "GPA of 3.5 or higher" $(n=51,32.3 \%)$ of a "GPA of 3.00 to 3.49 ," and ( $n=16,10.1 \%)$ of "GPA 3.00 and under." When the participants were asked what type of residence they currently reside in, $55.7 \%$ of participants live "on-campus" and $44.3 \%$ live "off-campus." Sixty-three of the total participants (39.9\%) reported that they do not get a sufficient amount of sleep each night. Ninety-five participants (60.1\%) reported that they do not get a sufficient amount of sleep each night. While only $(n=28,17.7 \%)$ of the participants have a suspicion that they have a sleeping disorder.

The participants were asked what sleep environment variables apply to them and the responses consisted of "bed sharing" $(n=20,12.7 \%)$, "room sharing" $(n=68,43 \%)$, "bedroom intrusive noise" ( $n=48,30.4 \%)$, "bedroom intrusive light" ( $n=38,24.1 \%)$, "temperature too high" ( $n=34,21.5 \%)$, "temperature too low" $(n=21,13.3 \%)$, and "none" $(n=38$, 24.1\%).The three responses to the "other" item in this section are as follows: "sleep in the living room," "roommate is too moody," and "house sharing." This information is depicted below in Figure 1. Sleep Environment Variables.

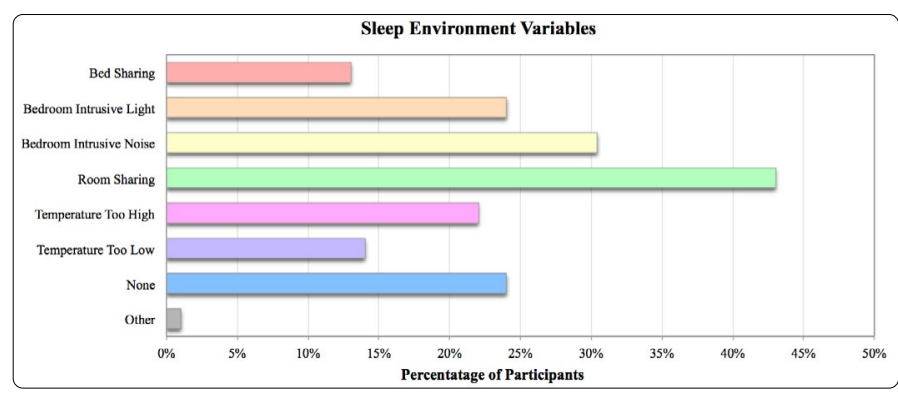

Figure 1. Sleep Environment Variables.

Every participant in the study reported being affected by daytime sleepiness to some degree. When asked how often the participant experiences daytime sleepiness the reports included the following: $(n=59,37.3 \%)$ "occasionally," $(n=82$, $51.9 \%)$ "frequently," and ( $n=17,10.8 \%)$ "always." The participants were then asked how often their daytime sleepiness affects their academic performance and the responses included the following: $(n=0,0 \%)$ "no daytime sleepiness," $(n=50,31.6 \%)$ "never," $(n=22,13.9 \%)$ "monthly," $(n=62,39.2 \%)$ "weekly," and $(n=21,13.3 \%)$ "daily."The participants were asked if they are on a regular sleep schedule (falling asleep and waking up at the same time) and the reports included the following: $(n=12,8 \%)$ "never," $(n=60$, $38 \%)$ "sometimes," $(n=69,43.7 \%)$ "during the week," $(n=1$, $1 \%)$ "on the weekend," and ( $n=16,10.1 \%)$ "always."

When participants were asked which of the following affect their quality of sleep, the responses consisted of the following: $(n=120,76 \%)$ "stressful lifestyle," $(n=101,64 \%)$ "class schedule," ( $n=99,62.7 \%)$ "course workload," $(n=55$, $34.8 \%)$ "work schedule," $(n=54,34.2 \%)$ "caffeine," $(n=22$, $13.9 \%)$ "physiological condition," ( $n=14,9 \%)$ "alcohol," $(n=$ $12,8 \%)$ "medication," $(n=5,3 \%)$ "nicotine," $(n=6,4 \%)$ "none," and $(n=6,4 \%)$ "other." The five responses to the "other" item in this section are as follows: "pregnancy," "sorority schedule," "social media," "night terrors," and "being in love." This information is depicted below in Figure 2. Variables affecting Quality of Sleep.

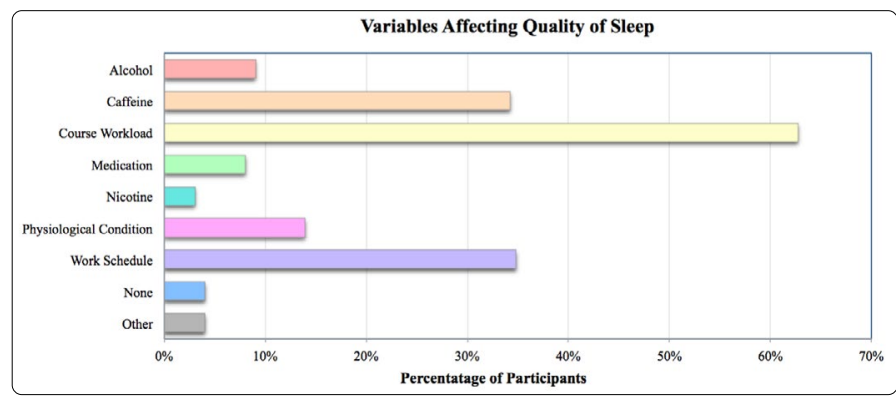

Figure 2. Variables Affecting Quality of Sleep

When participants were asked about their sleep duration the night before an exam, the responses consisted of: $(n=22$, $13.9 \%)$ "much less," ( $n=50,31.6 \%)$ "less," $(n=81,51.3 \%)$ "average," $(n=5,3 \%)$ "more," and $(n=0,0 \%)$ "much more."When the students were asked what sleep aids they currently use and the responses included the following: $(n=80,50.6 \%)$ "none," $(n=24,15.2 \%)$ "melatonin," $(n=23,14.6 \%)$ "regular exercise," ( $n=18,11.4 \%)$ "deep breathing," $(n=18,11.4 \%)$ "soothing music," ( $n=13,8.2 \%)$ "chamomile," $(n=12,8 \%)$, 
"muscle relaxation," ( $n=9,6 \%)$ "meditation," ( $n=3,2 \%)$ "sleep diary," ( $n=3,2 \%)$ "valerian," and $(n=2,1 \%)$ "guided imagery." This information is depicted below in Figure 3. Sleep Aids.

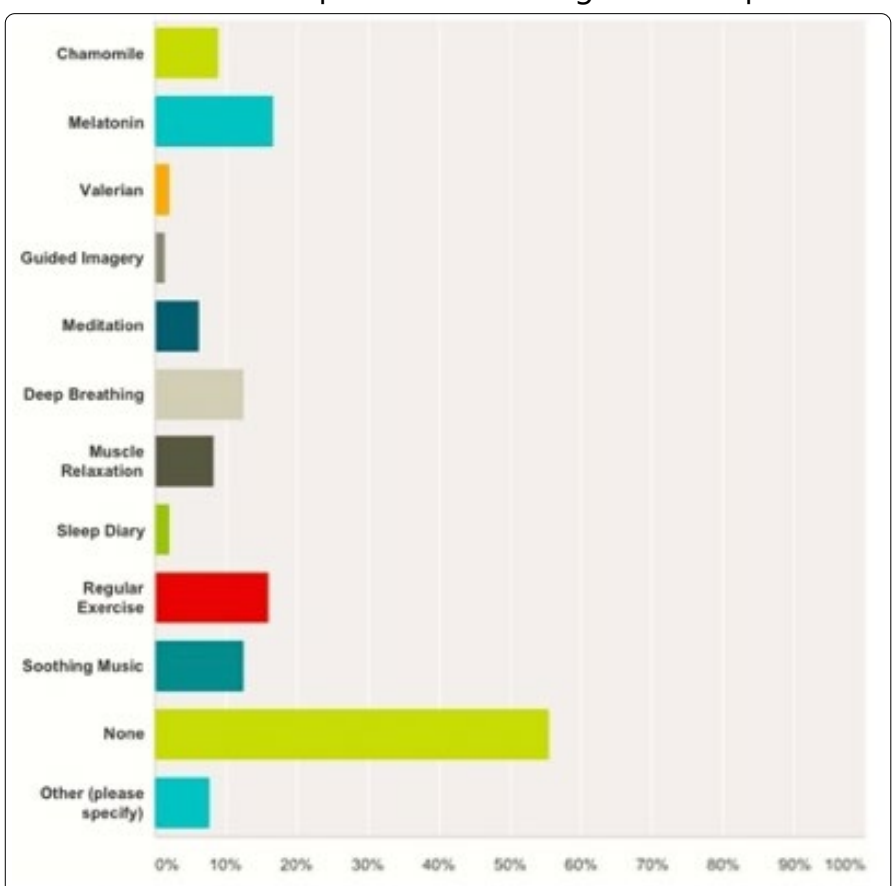

Figure 6: Sleep Aids

Figure 6. Sleep Aids

\section{GPA and Sleep Duration the Night before an Exam}

GPA was related to sleep duration the night before an exam. The independent variable was the sleep duration the night before an exam (compared to a typical night) measured among the following four categories: more, average, less, and much less. GPA was measured among four categories including the following: GPA of 3.50 or higher, GPA of 3.00 to 3.49, and GPA under 3.00. The data were analyzed using the $\chi^{2}$ test of independence. The relation between these variables was statistically significant with a $p$-value of $=0.03$. See Table 1 below for more details.

\begin{tabular}{|c|c|c|c|c|c|c|}
\hline & \multicolumn{7}{|c|}{ Hours of Sleep } \\
\hline GPA & More & Average & Less & Much Less & NA & Total \\
\hline GPA of 3.50 or higher & 0 & 56 & 25 & 8 & 2 & 91 \\
\hline GPA of 3.00 to 3.49 & 4 & 20 & 20 & 7 & 0 & 51 \\
\hline GPA under 3.00 & 1 & 5 & 5 & 5 & 0 & 16 \\
\hline$x=13.744$ & $\mathrm{df}=6$ & \multicolumn{7}{|c|}{ p-value $=0.03263$} \\
\hline
\end{tabular}

Table 1. $\chi^{2}$ test of independence regarding GPA and sleep duration the night before an exam

\section{GPA and Mean Sleep Duration on a Typical Night}

Sleep duration on a typical night was compared to GPA. A self-report length from 1 hour to 10 hours measured the independent variable of sleep duration on a typical night. The data were analyzed using a one-way ANOVA in Table 2 below. The higher the reported GPA, the longer the sleep duration the student received on a typical night.

\begin{tabular}{|c|c|c|c|c|}
\hline GPA & Mean Sleep Duration & SD & Data: $n$ & Data: NA \\
\hline GPA of 3.50 or higher & 6.967391 & 0.9994623 & 92 & 1 \\
\hline GPA of 3.00 to 3.49 & 6.468085 & 0.905324 & 47 & 0 \\
\hline GPA under 3.00 & 6.200000 & 1.5212777 & 15 & 1 \\
\hline
\end{tabular}

Table 2. One-way ANOVA over GPA and mean sleep duration on a typical night

\section{Number of Courses and Frequency of Daytime Sleepiness}

The number of courses was compared to frequency of daytime sleepiness in university students. The independent variable of number of courses was measured using three categories including: 1-3 courses, 4 courses, and 5+ courses. The categories of one course through three courses were consolidated due to the limited number of responses for the categories of one, two, or three courses. The dependent variable of frequency of daytime sleepiness was measured using three categories including the following: "occasionally," "frequently" and "always". The category of "never" was removed during statistical analysis due to none of the participants selecting this option. The category "rarely" was consolidated into the "occasionally" category because only four participants selected this option. The data were analyzed using the $\boldsymbol{\chi}^{2}$ test of independence. The relationship between these variables was significant with $p=0.04$. Participants who were taking more courses reported a higher frequency of daytime sleepiness. The results are outlined in Figure 3 below.

\begin{tabular}{|c|c|c|c|}
\hline & \multicolumn{3}{|c|}{ Frequency of Daytime Sleepiness } \\
\hline Number of Courses & Occasionally & Frequently & Always \\
\hline 1 -3 Courses & 12 & 6 & 2 \\
\hline 4 Courses & 16 & 26 & 3 \\
\hline $5+$ Courses & 50 & 27 & 12 \\
\hline \multicolumn{2}{|c|}{ X-squared $=10.0084$} & $\mathrm{df}=4$ & \multicolumn{2}{c|}{$\mathrm{p}$-value $=0.04029$} \\
\hline
\end{tabular}

Table 3. $\chi^{2}$ test of independence regarding number of courses and frequency of daytime sleepiness

\section{Discussion}

The principle aim of this study was to assess the relationship between the various influences that affect quality of sleep and how academic performance is thus influenced in university students at a midsize rural university. Poor quality of sleep, including short sleep duration, irregular sleep schedules, sleep deprivation, and daytime sleepiness, have all been indicated to negatively affect university students' academics by (Brown et al., 2006; Chen et al., 2014; Hershner $\&$ Chervin, 2014; Giannotti et al., 2002). In this study the data reflect similar results, demonstrating a statistical significance between sleep duration and cumulative GPA, and number of courses enrolled and frequency of daytime sleepiness.

The trend from Table 1 is that participants with a higher cumulative GPA tended to get the same or more hours of sleep the night before an exam compared to a typical night's sleep duration. Participants with lower cumulative GPAs tended to have shorter sleep duration the night before an 
exam. A possible explanation to account for this statistically significant difference between GPA and sleep duration the night before and exam is that students who have a high GPA tend to study more throughout the week and well before exams occur. Participants with a lower GPA procrastinate studying and then they attempt to pull an "all-nighter" the night before the exam; they are illustrated in the lower GPA of participants that suffer from acute sleep deprivation. Giannotti et al. (2002) supports that acute sleep deprivation affects academic performance negatively [10-15].

The results from Table 2 illustrate the sleep duration of a participant on a typical night of sleep compared to their GPA. Participants with a GPA of 3.50 or higher reported sleep duration on average of 6.97 hours, while participants with a GPA between 3.00 and 3.49 reported a GPA of 6.47 hours, and participants with a GPA under 3.00 get a sleep duration of 6.20 hours on average. These results support the proposed hypothesis that students who have longer sleep duration have better academic performance. Poor sleep quality is associated with poorer school performance (Brown et al., 2006; Chen et al., 2014; Hershner \& Chervin, 2014) which supports the findings of this study related to sleep duration. The measure of the participants' academic profiles in this current study included only their cumulative GPA. GPA is a measurement of more than just academic performance in university students; GPA also measures the ability of the student to effectively manage the complex interaction of influences in the student's environment (Orzech et al., 2011).Students are able to better manage their time with the various social, academic, and familial commitments that may occur throughout the semester and still manage to study and get an adequate amount of sleep each night.

The Chi-squared test for independence used on Table 3 results for the independent variable of number of courses enrolled and dependent variable of frequency of daytime sleepiness demonstrated the statistical significance that students enrolled in more courses are more likely to have an increased frequency of daytime sleepiness. Milner and Cote (2009) stated that university students are more likely to suffer from daytime sleepiness from a naturally delayed circadian rhythm. If the student develops a delayed circadian rhythm, the student will not become sleepy until later in the night, but will still have to be awake for early classes. Hershner and Chervin (2014) also support this finding that circadian rhythms and the homeostatic sleep drive affect the amount of daytime sleepiness students experience. Participants who are enrolled in more courses, or courses with more strenuous academic demand, are more likely to stay up longer to work on academics. Shifting to later bedtimes leads to later rise times, missing early morning classes, decreased awareness and lower grades (Sexton-Radek, \& Hartley, 2013). This suggests that university students should limit their academic demand to a manageable amount so that sufficient sleep can still be achieved with enough time to be adequately prepared for their classes.

When participants were asked about what variables affect their sleep, stressful lifestyle, class schedule, course workload, work schedule, and caffeine were the top five variables that were reported. The participants were not asked whether or not they had a job at any point in this survey, but work schedule was a variable that affected 55 participants' duration of sleep. This study did not account for such various time commitments that demand a student's time other than academics and duration of sleep. Similar to the nursing students in the Ferreira and Martino (2012) study, many participants in this study are trying to juggle a job, course requirements on top of class lectures, and the use of caffeine and medication to modify their sleep patterns. The participants in this study also recognized variables that were not expected to affect quality of sleep. The variables included the following: "sorority schedule," "social media," and "being in love." Orzech et al. (2011) recognized that students experience many variables in their daily lives, with some students being away from home for the first time, that affect their duration of sleep. The age and developmental level of university students supports this finding in that students are building a social identity and interacting constantly with others to form close bonds.

In the questionnaire, participants were asked about their use of sleep aids to manipulate their sleeping schedule. The results, in Figure 3, illustrated a surprising amount of students, $50.6 \%$, who reported that they do not use any type of sleep aid. In addition, $60.1 \%$ of the participants reported that they do not get a sufficient amount of sleep, and $52.5 \%$ believe that their daytime sleepiness affects their academic performance. Brown et al. (2006) states that many students do not know what constitutes good sleep habits or the impact of sleep duration on other aspects of their lives. There is a lack of knowledge and education about sleep aids and their effectiveness in enhancing or hindering quality of sleep. For instance, the students may not know that behavioral modifications such as regular exercise, meditation, and deep breathing are interventions that can enhance quality of sleep. Increasing student's knowledge about the positive effects of the available sleep aids can naturally enhance their quality of sleep. Medication and caffeine that participants report consuming can affect the student's body in a way that the circadian rhythm and the homeostatic sleep drive do not encourage the student to sleep during their normal sleeping hours. Behavioral modifications to aid in falling asleep may not be effective. Robinson et al. (2013) agree that students take medication and other herbal remedies to modify their sleep patterns to accommodate for the need for increased attention for their social, academic and employment obligations. Whether the medications are stimulants or depressants, the body may not recognize the need for sleep from hormone levels and normal body processes, and the body can eventually become dependent on the medication or caffeine for the student to reach the desired states of consciousness.

The sleep environment of university students is unique in that it contains more intrusive variables from the shared living environment than the sleep environment of other adults. Of the 88 participants of this study who lived on-campus, 68 participants reported room sharing, which makes sense since most of the housing on-campus requires room sharing. 
Bedroom intrusive noise reported by 48 participants, and bedroom intrusive light reported by 38 participants also coincide with both on-campus and off-campus housing due to multiple university students living within than same residence, but not necessarily the same room. Sexton-Radek and Hartley (2013) agree in that noise in a residential hall of university students was the common reported disturbance of students' sleep environments. The variables of noise, light, temperature, and bed/room sharing in the Chen et al. (2014) study support this finding. All of the sleep environment variables can hinder a student's duration of sleep. Many students report the effect of multiple variables affecting their overall sleep quality. The more variables experienced by the student through the night, the less likely they are able to get a good night's sleep before class the next day, which could impair their attention and concentration, and overall grade point average, a direct measurement of academic performance.

The researcher proposed to investigate the need of implementing a sleep education program component to improve the overall quality of sleep in university students. A sleep education program would teach students how sleep hygiene can be used to improve daily sleep habits by counseling the student to go to bed and wake up at the same time each day, avoid consuming caffeine and other stimulants in the late afternoon and evening, monitor nighttime eating, and engage in relaxing activities before bed, such as reading or listening to soothing music. "To understand the consequences of sleepiness and sleep deprivation, knowledge of normal sleep and its impact on learning, memory, and performance are necessary" (Hershner and Cherivn, 2014, p. 674).

This study was relevant for utilization in academic settings because of the statistically significant evidence that duration of sleep is related to academic performance. The dissemination of information on the importance of sleep hygiene is essential to improve the overall quality of sleep in university students. Implementation of interventions to promote better sleep quality can improve student's grades and increased the likelihood of finding a career after college (Shochat et al., 2014).

\section{Suggestions for Further Research}

Further research could examine the napping habits of university students. Questions about the topic of napping were omitted from the questionnaire. The questions could examine napping habits such as what time during the day and for how long the student takes a nap and whether the nap is beneficial in relieving daytime sleepiness. Also, further investigation of which chronotype of circadian preference the participants identify with, either morning (morning person) or evening (night owl), can be helpful in determining whether the participant's attention span in early morning classes are affected by the participants natural circadian rhythm. Students could also be asked whether or not they partake in any type of behavior that stimulates the central nervous system such as drinking caffeine or working out within hours before attempting to sleep. Asking the students whether or not they have a job or how long they are able to study each day can affect the measurement of the student's academic profile.

A longitudinal study would provide insight on sleep habits of university students throughout the semester or academic year. Academic demands change throughout semester, with final exams and other various course requirements due toward the end of the semester. University students may need continued education and reminders to practice healthy hygiene toward the end of the semester when academic demand is increased. Another study could include a sleep diary to collect a more accurate sleep profile of each participant as well. The sleep diary would allow the participant to record the exact times when the student goes to bed and wakes up in the morning, the sleep environment variables affected falling asleep each night, and how many times he/ she wakes up throughout the night.

\section{Limitations}

The convenience of electronic administration of the questionnaire may have been a factor in not obtaining a representative sample of the student population. The administration of the questionnaire to include honors program students could have skewed the distribution in favor of a higher GPA. The question in the survey that asked participants what their cumulative GPA is also based on selfreport from the participant, therefore students may have fabricated their answer. Also, the honors program at the university is mostly made up of female students, which would also explain the high number of female participants (85\%) in this study, even though the student population at the university is sixty percent female.

A limitation of the research study is that the sample is not very diverse. A very low proportion of students who participated in the study are in various ethnic groups. The study was unable to analyze a difference among ethnic groups because the sample was relatively homogenous when related to ethnicity, demonstrating the predominately white/ Caucasian population on the campus. The sample size of 158 participants is small compared to the population of 11,000 students enrolled at the university where the study was conducted. Due to this small sample size, the data and inferences in this study may not be generalizable to all universities of the same size.

Data were disregarded if the participant stated he or she was under the age of eighteen at the beginning of the questionnaire. Only two respondents fell into this category. Participants left eight questionnaires incomplete and their responses were omitted from the final results analysis as well.

The variables in this study did not assess the participant's academic performance as accurately as possible. Instead of asking the participants how many courses they were currently taking, it may have been more accurate to ask the participants how many credit hours in which they were enrolled. Also, the questionnaire should have defined levels of scholarship as 
they pertain to 100-, 200-, 300-, 400-, and 500-level courses. Defining the distinctions between lower and upper level curricula may help to inform the researcher on the difficulty of the course curriculum. For instance, graduate students take fewer courses, but the courses are all higher level of scholarship. Lastly, since this study was a cross-sectional study, it may have been more accurate to measure academic performance in the form of final grades instead of overall cumulative GPA.

\section{Implications}

Using the results of the study, students could be taught what in a school setting it means to have healthy sleep hygiene. By doing this, students are made aware of the habits they need to obtain to increase overall quality of sleep. An overview of sleep hygiene skills would also be taught as a sleep education component in everyday curriculum. It would introduce active sleep hygiene skills, such as daily exercise. Also, the education component would include non-active sleep hygiene skills, such as creating a calm environment when trying to fall asleep. By introducing these skills, the students will become more aware of the various types of sleep hygiene skills, and how these skills can help reduce daytime sleepiness and positively affect their ability to learn.

The students would continue to be educated about sleep hygiene skills. But in addition to that, they would be taught the physiological signs and symptoms of sleep deprivation so that they can identify when their quality of sleep is not at its best. An education piece about influences that negatively impact sleep such as avoiding caffeine consumption in the evening hours and not studying while lying in bed would also be included on this sleep hygiene educational program. Students can elect to practice all of these skills, or only skills they find are beneficial to promote their own healthy sleeping habits.

Application of this recommendation would include educating parents and schoolteachers and professors about the potential sleep hygiene skills that can be used to improve quality of sleep so that they can encourage the students and children around them to practice healthy habits. Integration of an educational program for students beginning in their middle school years is crucial to begin healthy sleeping habits at an early age, rather than change unfavorable sleeping habits at a later age. With more students educated on the aspects of healthy sleep hygiene, there is a potential for students to learn more efficiently and achieve more in the scope of academics.

\section{Conclusion}

This study has illustrated a connection between the duration of sleep and academic performance in students. Healthy sleeping habits of a regular sleep schedule with adequate sleep duration each night is beneficial to students and their academic achievement. The need of implementing a sleep education program component to students has been identified. The program would consist of teaching students various aspects of a healthy sleep hygiene, such as, to fall asleep and wake up at the same time throughout the week, to partake in daily exercise but to engage in relaxing activities before bed, not to drink caffeine in the evening, and the many other aspects included in healthy sleep hygiene. An educational program such as this would not be diffuclt to implement due to similar programs that are already in place in schools that educate students over heathly eating habits. In addition to school nutrition programs,schools should include in their curriculum a program teaching healthysleep hygiene to promote a healthy physical state that is favorable for learning.

Conflicts of Interest: The authors declare no conflicts of interest with this submission.

\section{References}

1. Bahammam AS, Alaseem AM, Alzakri AA, Almeneessier AS, Sharif MM The relationships between sleep and wake habits and academic performance in medical students: A cross-sectional study. BMC Medical Education, 2012; 1261. doi: 10.1186/1472-6920-12-6

2. Franklin $C B$, Walter $C B$, Barlow $S$. Development and evaluation of the sleep treatment and education program for students (STEPS). Journal Of American College Health, 2006; 54(4), 231-237. doi: 10.3200/ JACH.54.4.231-237

3. Chen T, Wu Z, Shen Z, Zhang J, Shen X, Li S. Sleep duration in Chinese adolescents: biological, environmental, and behavioral predictors. Sleep Medicine, 2014; 15(11), 1345-1353. doi: 10.1016/j.sleep.2014.05.018

4. Ferreira LRC, Martino MMFD. Sleep patterns and fatigue of nursing students who work. Revista da Escola de Enfermagem da USP, 2012 46(5), 1178-1183.

5. Fox J. The R commander: A basic statistics graphical user interface to $R$. Journal of Statistical Software, 2005; 14(9), 1-42.

6. Giannotti F, Cortesi F, Sebastiani T, Ottaviano S. Circadian preference, sleep and daytime behaviour in adolescence. Journal Of Sleep Research, 2002; 11(3), 191-199. doi: 10.1046/j.1365-2869.2002.00302.x

7. Gomes AA, Tavares J, \& de Azevedo MP. Sleep and academic performance in undergraduates: a multi-measure, multi-predictor approach. Chronobiology International, 2011; 28(9), 786-801. doi: 10.3109/07420528.2011.606518

8. Hershner SD, Chervin RD. Causes and consequences of sleepiness among college students. Nature and Science of Sleep, 2014; (6) 73-84. doi: 10.2147/NSS.S62907

9. Lovato $\mathrm{N}$, Lack $\mathrm{L}$, Wright $\mathrm{H}$. The napping behaviour of Australian university students. Plos One, 2014; 9(11), e113666. doi: 10.1371/journal. pone. 0113666

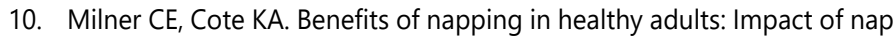
length, time of day, age, and experience with napping. Journal Of Sleep Research, 2009; 18(2), 272-281. doi: 10.1111/j.1365-2869.2008.00718.x

11. Orzech KM, Salafsky DB, Hamilton LA. The state of sleep among college students at a large public university. Journal Of American College Health 2011; 59(7), 612-619. doi: 10.1080/07448481.2010.520051

12. Robinson $D$, Gelaye $B$, Tadesse MG, Williams MA, Lemma $S$, Berhane $Y$ Daytime sleepiness, circadian preference, caffeine consumption and Khat use among college students in Ethiopia. Journal of Sleep Disorders Treatment \& Care, 2013; 3(1) doi: 10.4172/2325-9639.1000130

13. Sexton-Radek K, Hartley A. College residential sleep environment Psychological Reports, 2013; 113(3), 903-907.

14. Shochat $T$, Cohen-Zion M, Tzischinsky O. Functional consequences of inadequate sleep in adolescents: A systematic review. Sleep Medicine Reviews, 2014; 18(1), 75-87. doi: 10.1016/j.smrv.2013.03.005

15. Walker MP, Brakefield T, Morgan A, Hobson JA, Stickgold R. Practice with sleep makes perfect: Sleep-dependent motor skill learning. Neuron, 2002; 35(1), 205-211. 


\section{Appendix}

Quality of Sleep and Academic Performance in University Students

Please review the questionnaire and ask any questions you have before beginning the survey. You may keep the cover letter for your records. Once you have finished, please return the questionnaire to the primary investigator.

1. Age:

2. What is your gender: Male Female

3. What is your ethnicity? (Please select all that apply)

American Indian or Alaskan Native Asian or Pacific Islander

White / Caucasian Prefer not to Answer

Black or African American

Other:

Academic Evaluation

4. Student classification at the institution:
Freshman

$$
\text { Sophomore }
$$

Junior

Senior

Graduate

5. How many courses are you taking?

$$
1 \text { Course } 2 \text { Courses }
$$

3 Courses

4 Courses

$5+$ Courses

6. When do your classes normally begin each day?

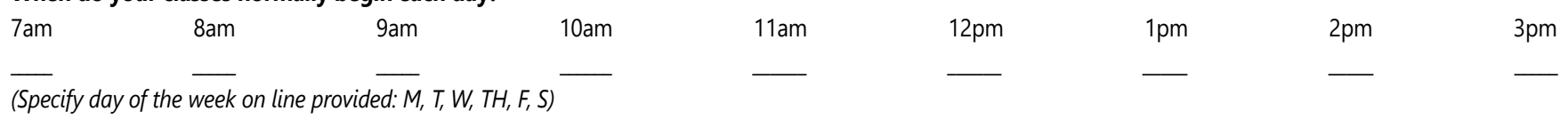

7. What is your cumulative GPA?

GPA of 3.50 or higher (Mostly As) SEEP?

GPA of 2.50 to 2.99 (Some Bs and Cs)

GPA of 3.00 to 3.49 (Mostly Bs)

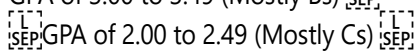

GPA less than 2.00 (Lower than Cs)

Sleep Quality Evaluation

8. What is your current type of residence?

On-Campus

Off-Campus

9. Sleep environment variables that apply to you: (Please select all that apply)

$\begin{array}{ll}\text { Bed sharing } & \text { Room sharing } \\ \text { Bedroom intrusive light } & \text { Temperature too high } \\ \text { None } & \end{array}$

None

Bedroom intrusive noise

Temperature too low

Other:

10. How many hours of sleep do you get on average during any given night?
1
23
$3 \quad 4 \quad 5$
6
7
8
9
10
$10+$

11. Do you feel that you get a sufficient amount of sleep?

Yes

No

12. Do you have any suspicion that you may have a sleeping disorder?

Yes

No

13. How many times do you wake up during the night on average?
0
2
3
4
56
7
8
9
10

14. How often do you experience daytime sleepiness?
Never
Rarely
Occasionally
Frequently
Always

15. How often do you believe your daytime sleepiness affects your academic performance?

No Daytime Sleepiness Never Daily

Weekly

Monthly

16. Are you on a regular sleep schedule (falling asleep and waking up at the same time)?
Never
Sometimes
During the Week

On the Weekends

Always

17. Do you think any of the following may have an impact on your quality of sleep? (Please select all that apply)

$\begin{array}{llll}\text { Medication } & \text { Stressful Lifestyle } & \text { Work Schedule } & \text { Class Schedule } \\ \text { Course Workload } & \text { Psychological Condition } & \text { Caffeine } & \text { Nicotine } \\ \text { Alcohol } & \text { None } & \text { Other: } & \end{array}$

18. On a night before an exam, rate the amount of sleep hours compared to normal:
Much Less
Less
Average
More
Much More

19. Do you use any of the following sleep aids (Circle all that apply)?
Chamomile
Melatonin
Valerian
Muscle Relaxation
Sleep Diary
Regular Exercise
Guided Imagery Meditation Deep Breathing
Soothing Music
None
Other: 